\title{
Pediatric Medical Device Terminology
}

National Cancer Institute

\section{Source}

National Cancer Institute. Pediatric Medical Device Terminology. NCI Thesaurus. Code C122636.

Terminology developed by a collaborative effort of the National Cancer Institute's Enterprise Vocabulary Services, the National Institute of Child Health and Human Development, and an international group of clinicians and informaticists to support the use of medical devices in the pediatric population. 